bioRxiv preprint doi: https://doi.org/10.1101/444539; this version posted October 16, 2018. The copyright holder for this preprint (which was not certified by peer review) is the author/funder, who has granted bioRxiv a license to display the preprint in perpetuity. It is made available under aCC-BY-NC-ND 4.0 International license.

\title{
1 Rhizosphere microbes influence host circadian clock function
}

2 Charley J. Hubbard ${ }^{1,2}$, Robby McMinn ${ }^{1,2}$, Cynthia Weinig ${ }^{1,2,3}$

3 1. Department of Botany, University of Wyoming, Laramie, WY, USA

4 2. Program in Ecology, University of Wyoming, Laramie, WY, USA

5 3. Department of Molecular Biology, University of Wyoming, Laramie, WY, USA 


\section{Abstract}

8 The circadian clock is an important determinant of individual fitness that is entrained by local

9 conditions. In addition to known abiotic inputs that entrain the circadian clock, individual

10 pathogenic soil bacteria affect the circadian period of plant hosts. Yet, in nature, plants interact

11 with diverse microbial communities including hundreds to thousands of microbial taxa, and the

12 effect of these communities on clock function remains unclear. In Arabidopsis thaliana, we used

13 diverse rhizosphere inoculates and both wild-type and clock mutant genotypes to test the effect

14 of complex rhizosphere microbial communities on the host circadian clock. Host plants with an

15 intact rhizosphere microbiome expressed a circadian period that was closer to 24 hrs in duration

16 and significantly shorter (by 60 minutes on average) relative to plants grown with a disrupted

17 microbiome. Wild-type host genotypes differed significantly in clock sensitivity to microbiome

18 treatments, where the effect was most pronounced in the Landsberg erecta genotype and least in

19 the Columbia genotype. Rhizosphere microbes collected from a host genotype with a short-

20 period phenotype (toc $1-21$ ) and used as inoculate significantly shortened the long-period

21 phenotype of the $z t l-1$ clock mutant genotype. The results indicate that complex rhizosphere

22 microbial communities significantly affect host clock function. 


\section{Introduction}

25

26

27

Across all domains of life, organisms have evolved endogenous time-keeping mechanisms known as circadian clocks [1]. In plants, the clock regulates a large percentage of the transcriptome, the metabolome, leaf-level and organismal physiology, and even the composition of beneficial rhizosphere microbial communities [2-5]. There are several well-described abiotic and biotic factors leading to clock entrainment, including photoperiod, light intensity, temperature, and herbivory $[3,6]$. A recent study has shown that soil microorganisms may be another environmental factor that entrain the plant circadian clock [7]. Specifically, Zhang et al. (2013) found that Arabidopsis thaliana plants infected with Pseudomonas syringe had significantly shorter periods, that is, the duration of one circadian cycle, than uninfected plants. Zhang et al. (2013) demonstrate that individual microbial taxa affect clock function. In nature, plants interact with complex microbial communities consisting of hundreds to thousands of taxa [8]. Thus, the question remains as to how natural, complex microbial communities may influence clock function.

Microbiomes affect many aspects of host performance [8]. Although an organism's own genotype has been considered as the primary determinant of phenotype for many traits, there is growing evidence that host-associated microbial communities and their genomic functions also contribute to host phenotypes. In some cases, the phenotypic effects of microbial associates exceed the effects of host genetics [8,9]. For instance, studies of human-gut microbiome interactions have shown that the composition of gut microbial communities is a better predictor of host obesity than is host genetics [10]. The genetic pathways underlying clock function are well-described in plants, yet the magnitude of microbial effects $v s$. plant genotype on clock 
phenotypes is unknown. Comparing the effects of diverse inoculation treatments and host genotypes would reveal relative microbial $v s$. host genetic contributions to clock phenotypes.

In the current study, we test the effects of complex rhizosphere microbial communities on host clock function. We first characterize the effects of rhizosphere microbes on the host plant clock by comparing the circadian period of plants grown in intact $v s$. disrupted rhizosphere microbiome treatments. Next, we examine the relative effects of distinct microbial treatments $v s$. plant genotypes on circadian period. We then test if rhizosphere microbial communities found in association with host plants with short (20-hr) vs. wild-type (24-hr) circadian periods can rescue (shorten) the long-period phenotype (28-hr) of a mutant genotype with disrupted clock function.

\section{Materials and Methods}

$\underline{\text { Plant material and growth conditions }}$

To investigate the influence of rhizosphere microbes on host plant circadian period, we used Arabidopsis thaliana accessions that harbor the reporter gene LUCIFERASE (LUC), the gene responsible for bioluminescence in the presence of the substrate luciferin in fireflies (Photinus phralis), linked to the native circadian gene, COLD CIRCADIAN RHYTHM RNA BINDING 2

$62(C C R 2)$, allowing for quantification of circadian parameters [11]. Genotypes included in the current study are Columbia (Col), Strand (Nrw), Wassilewskija (Ws), Landsberg erecta (Ler), and both the short-period mutant TIMING OF CAB EXPRESSION 1 (toc1-21) and the longperiod mutant of ZEITLUPE (ztl-1) in the Col background. 
sucrose, and cold stratified for 7 days at $4^{\circ} \mathrm{C}$. To germinate and entrain plants, plates of planted seeds were placed in a Percival PGC-9/2 growth chamber (Percival Scientific, Perry, IA, USA) set on a 12 / 12 light / dark cycle at $22^{\circ} \mathrm{C}$. After 7 days, plants were inoculated with $10 \mu$ l of a microbial inoculant created combining $10 \mathrm{~g}$ of soil with $90 \mathrm{ml}$ of $\mathrm{ROH}_{2} \mathrm{O}$ and filtered through 1 000, 500, and 212 sieves to remove nematodes that could detrimentally affect plant performance [12]. 24 hours after inoculation, plates were moved into an ORCA-II ER digital camera (Hamamatsu Photonics, Bridgeport, NJ, USA) for imaging and quantification of bioluminescence.

Each 96 well plate contained 3 genotypes with 32 replicates each. Within each genotype there were 4 rhizosphere microbiome treatments with 8 replicates each. Rhizosphere microbiome treatments used in this study include: intact, disrupted, Columbia, and toc1-21. To generate the intact treatment, we used soil from the Catsburg site in North Carolina that has a welldocumented occurrence of wild A. thaliana plants [13]. For the disrupted treatment, intact inoculant was autoclaved or filtered through $0.2 \mu \mathrm{m}$ mesh to disrupt microbial communities while maintaining the nutrient load found in the intact treatment. The Columbia and toc1-21 rhizosphere microbiome treatments reflect rhizosphere microbial communities collected from the Col and toc1-21 A. thaliana genotypes (that had been initially treated with the Catsburg inoculant). The Col and toc1-21 rhizosphere treatments were included to determine if microbial communities might differ in their effects on clock period, and specifically if microbial communities found in association with a short-period mutant $v s$. wild-type host might differentially affect (shorten) the circadian period of the long-period mutant, $z t l-1$, which typically expresses a circadian period of $\sim 28 \mathrm{hr}$. All experiments were replicated 2-3 times. 


\section{$91 \quad$ Circadian Imaging}

92 Prior to imaging plants, $20 \mu \mathrm{l}$ of $100 \mathrm{mM}$ D-luciferin monopotassium salt and $0.01 \%$ Triton X-

93100 solution was added to each well. 30-minute-long exposure images were taken every hour for

94160 hours to quantify bioluminescence. Traces were then trimmed to $72 \mathrm{hr}$ windows and

95 manually inspected to ensure quality [14]. Traces that failed quality control were discarded. High

96 quality traces were then uploaded onto Biodare2 (https://biodare2.ed.ac.uk) to estimate circadian

97 period [15]. We used Two-Way ANOVAs to characterize the effects of rhizosphere microbiome

98 treatment, plant genotype, and their interaction on circadian period and Tukey's Honest

99 Significant Differences post hoc contrasts to characterize differences between genotypes and

100 rhizosphere microbiome treatments.

103 We characterized the influence of intact $v s$. disrupted rhizosphere microbiome treatments on the 104 period of two B. stricta genotypes with natural variation in clock period, genotype $15(\sim 22 \mathrm{hr}$ 105 period) and genotype 14 ( $23 \mathrm{hr}$ period) using the simulated fall entrainment conditions and 106 circadian leaf movement assays described in Salmela et al. 2016. 15 replicates of each genotype 107 were grown in each of two rhizosphere microbiome treatments, intact $v s$. disrupted, which were 108 generated using soil from the genotypes' home site (South Brush Creek, Wyoming, USA, 


\section{Results and Discussion}

114 The rhizosphere microbiome is often referred to as an extension of the plant genome, as complex

115 microbial communities affect many aspects of plant performance $[5,8,9]$. However, the extent to which complex rhizosphere microbial communities influence clock function remains unclear.

117 Here, we characterized the influence of the rhizosphere microbiome on host clock function. We 118 specifically addressed the questions, 1) do complex rhizosphere microbial communities influence 119 host circadian period? 2) What are the relative effects of the rhizosphere microbiome vs. plant genotype on circadian period? And 3) can rhizosphere communities shaped by plants with short (21-hr) or wild-type (24-hr) circadian periods rescue long-period mutant phenotypes?

122 Recent evidence suggests that infection with a single pathogenic microbial taxon, $P$. syringae

123 influences plant clock function through an effect on immune signaling [7]. We find that complex

124 communities of rhizosphere microbes significantly affect host plant circadian period (Figure 1).

125 Specifically, we find the circadian period is 60 minutes longer on average among plants grown

126 with a disrupted microbiome relative to plants grown with an intact microbiome, and the effect

127 of this clock deceleration is that period deviates increasingly from 24 hrs (Figure 1). Wild-type

128 genotypes (Col, Ler, Ws, and Nrw) differed significantly in the sensitivity of clock period to the 129 rhizosphere microbiome, with period being unaffected (Col) or changing by as much as 96 minutes (Ler). Likewise, in genotype 14 of B. stricta, we find that the period of plants grown in

131 disrupted treatment is up to 30 minutes longer than in plants grown in the intact treatment

132 (Figure 2). The largest observed changes in clock period could well affect plant fitness, because

133 the mismatch between endogenous and environmental cycle duration can significantly affect 134 performance [16-18] and in particular variation in clock period of this magnitude expressed in 135 near isogenic lines affects plant performance in the field [19]. Microbial associations with 
eukaryotes are, of course, evolutionarily ancient, and on average appear to modulate endogenous host circadian cycles, although some host genotypes appear more sensitive than others to the presence of microbes.

The 1.5-hour difference in circadian period observed for at least some genotypes (Ler) across the intact $v s$. disrupted rhizosphere microbiome treatments reflects a substantial portion of the range in circadian period expressed by the 4 wild-type $A$. thaliana accessions used in this study (50\%) and by a larger panel of 150 European accessions (23\%) [20]. Thus, rhizosphere microbes play an important role in clock function, but microbial effects appear of lesser magnitude than host genetic differences. As noted, there were significant interactions between rhizosphere microbiome treatment and plant genotype $(\mathrm{P}<0.001)$, where the period of some genotypes $(\mathrm{Col}$ in A. thaliana and 15 in B. stricta) were not affected by treatment, while the period of other genotypes (Ler, Nrw, Ws in A. thaliana and 14 in B. stricta) were more strongly responsive to rhizosphere microbiome treatments. Such genotype by rhizosphere microbe interactions suggest that there may be host genetic factors that permit microbes to affect the clock. A larger survey of diverse naturally occurring microbial communities and host genotypes will provide additional insights into the effect of microbial associates on host clock function.

\section{Compositional differences among intact rhizosphere microbiome treatments differentially} affected the circadian period of the long period clock mutant $z$ tl-1 (Figure 3). Here, ztl-1's period was significantly shortened when grown in the toc $1-21$ rhizosphere microbiome treatment compared to the disrupted rhizosphere microbiome treatment $(\mathrm{P}=0.016)$. To estimate circadian period using bioluminescence, plants are commonly grown in sterile media amended with antibiotics like kanamycin, suggesting that period estimates are made on plants either without rhizosphere bacterial associates or with severely disrupted communities $[17,19]$. As a result, 
159 such studies may not fully capture variability introduced by associated rhizosphere microbes, and

160 the variation among that exists among plant genotypes in natural settings. Further, although

161 rhizosphere microbiome treatments shaped by the Col and tocl-21 genotypes do not fully rescue

162 clock misfunction in $z t l-1$, the findings presented here suggest that rhizosphere legacy effects

163 could affect circadian period across generations.

164 In sum, we observed that complex microbial communities significantly influence host plant

165 clock function. Specifically, the effect of the rhizosphere microbiome on clock entrainment and

166 period length rivals that of differences among some plant genotypes and is equivalent to

167 moderate changes in well-described abiotic inputs, such as a 2-hour shift in photoperiod or

168 several degrees change in temperature $[1,3]$. Further, the effect of rhizosphere microbiomes on

169 circadian clock period is likely sufficient to alter plant performance, based on prior studies

170 testing the effects of quantitative clock variation on plant performance $[17,19]$. Given the

171 pronounced effects intact microbes have on the plant clock, future work should attempt to

172 identify the specific taxa and mechanisms by which microbes influence circadian period.

\section{Acknowledgments}

175 This work was supported by the National Science Foundation grants IOS-1444571 and EPS-

1761755726 to C.W. We would like thank Matthew J. Rubin and Jenney Moua for their help on this

177 project. 


\section{References}

181 1. McClung CR. Plant circadian rhythms. Plant Cell 2006; 18: 792-803.

182 2. Covington MF, Maloof JN, Straume M, Kay SA, Harmer SL. Global transcriptome analysis reveals circadian regulation of key pathways in plant growth and development. Genome Biol 2008; 9: R130.

3. Greenham K, McClung CR. Integrating circadian dynamics with physiological processes in plants. Nat Rev Genet 2015; 16: 598-610.

4. Yarkhunova Y, Edwards CE, Ewers BE, Baker RL, Aston TL, McClung CR, et al. Selection during crop diversification involves correlated evolution of the circadian clock and ecophysiological traits in Brassica rapa. New Phytol 2016; 210: 133-144.

5. Hubbard CJ, Brock MT, Diepen LT van, Maignien L, Ewers BE, Weinig C. The plant circadian clock influences rhizosphere community structure and function. ISME Journal,

7. Zhang C, Xie Q, Anderson RG, Ng G, Seitz NC, Peterson T, et al. Crosstalk between the circadian clock and innate immunity in Arabidopsis. PLoS Pathog 2013; 9: e1003370.

8. Bulgarelli D, Schlaeppi K, Spaepen S, van Themaat EVL, Schulze-Lefert P. Structure and health. Trends Plant Sci 2012; 17: 478-486. 
201 10. Sze MA, Schloss PD. Looking for a Signal in the Noise: Revisiting obesity and the microbiome. MBio 2016; 7: e01018-16.

11. Millar AJ, Short SR, Chua NH, Kay SA. A Novel Circadian Phenotype based on firefly luciferase expression in transgenic plants. Plant Cell 1992; 4: 1075-1087.

12. van de Voorde TFJ, van der Putten WH, Bezemer TM. Soil inoculation method determines the strength of plant-soil interactions. Soil Biol Biochem 2012; 55: 1-6.

13. Mauricio R, Rausher MD. Experimental manipulation of putative selective agents provides evidence for the role of natural enemies in the evolution of plant defense. Evolution (N Y) 1997; 51: 1435.

14. Millar AJ, Carré IA, Strayer CA, Chua NH, Kay SA. Circadian clock mutants in Arabidopsis identified by luciferase imaging. Science 1995; 267: 1161-3. period estimation methods for circadian data. PLoS One 2014; 9: e96462.

16. Salmela MJ, Greenham K, Lou P, McClung CR, Ewers BE, Weinig C. Variation in circadian rhythms is maintained among and within populations in Boechera stricta. Plant Cell Environ 2016; 39: 1293-1303.

217 17. Rubin MJ, Brock MT, Davis AM, German ZM, Knapp M, Welch SM, et al. Circadian rhythms vary over the growing season and correlate with fitness components. Mol Ecol 
19. Rubin MJ, Brock MT, Baker RL, Wilcox S, Anderson K, Davis SJ, et al. Circadian

20. Michael TP, Salomé PA, Yu HJ, Spencer TR, Sharp EL, McPeek MA, et al. Enhanced 


\section{Figures}

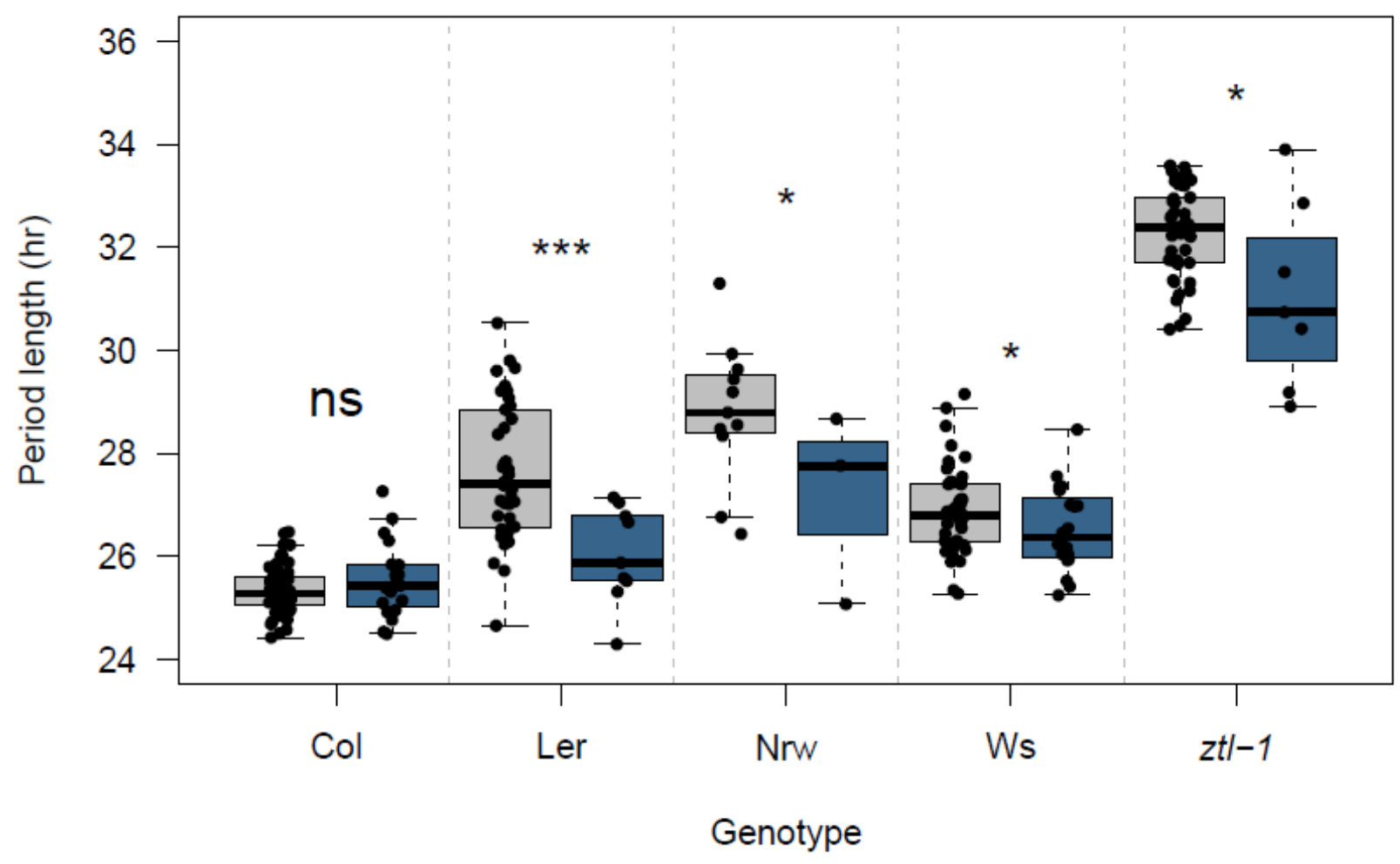

243 Figure 1: Rhizosphere microbiome treatment, intact (blue) vs. disrupted (gray), significantly

244 alters plant circadian period $(\mathrm{P}<0.001)$. Similarly, plant genotype $(\mathrm{P}<0.001)$ and interactions

245 between rhizosphere microbiome treatment and plant genotype $(\mathrm{P}<0.001)$ significantly affect

246 plant circadian period. The top and bottom of boxes represent the 75 th and 25 th percentiles,

247 respectively. Whiskers represent 1.5 times the interquartile range. Asterisks denote significant

248 differences between intact $v s$. two disrupted rhizosphere microbiome treatments within a given

249 genotype: $\mathrm{P}<0.05 *, \mathrm{P}<0.001 * * *, \mathrm{~ns}=$ non-significant 


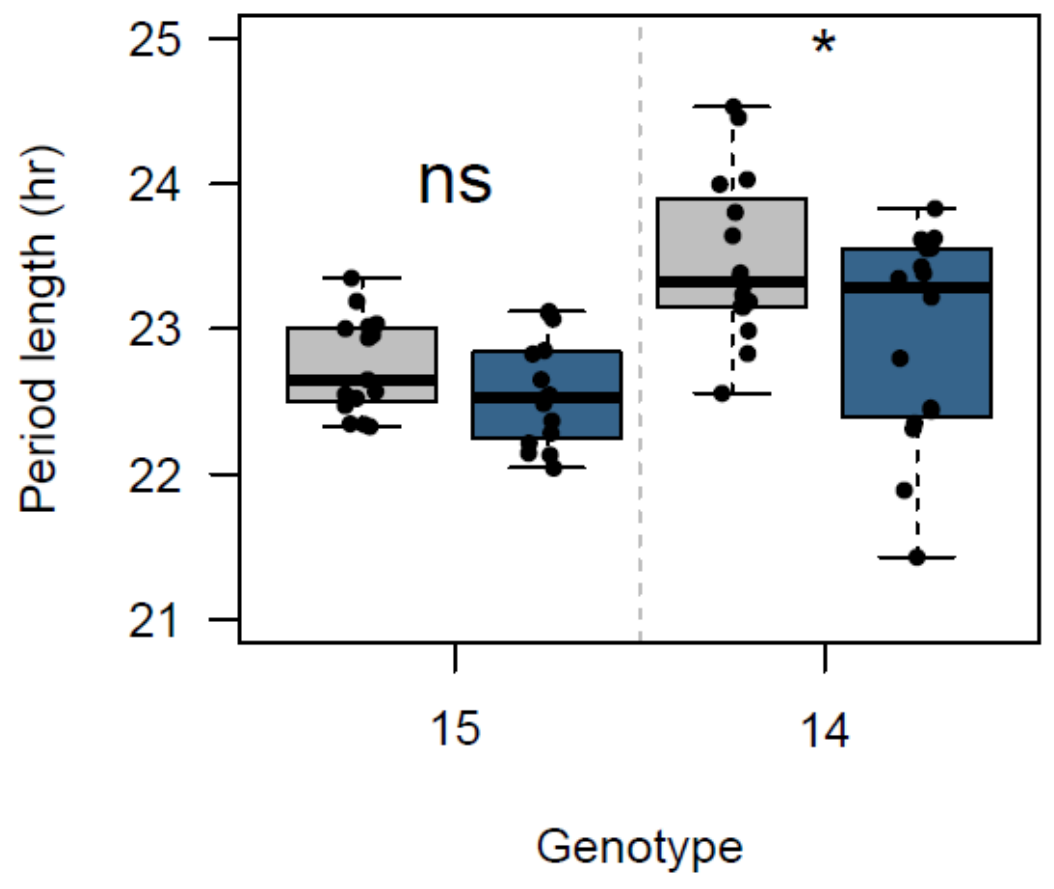

252 Figure 2: Rhizosphere microbiome treatment, intact (blue) vs. disrupted (gray), significantly

253 alters the circadian period of genotype $14(\mathrm{P}=0.032)$. Similarly, plant genotype significantly

254 affected plant circadian period $(\mathrm{P}<0.001)$. The top and bottom of boxes represent the 75 th and

25525 th percentiles, respectively. Whiskers represent 1.5 times the interquartile range. Asterisks

256 denote significant differences between intact $v s$. two disrupted rhizosphere microbiome

257 treatments within each genotype: $\mathrm{P}<0.05 *$, ns $=$ non-significant 


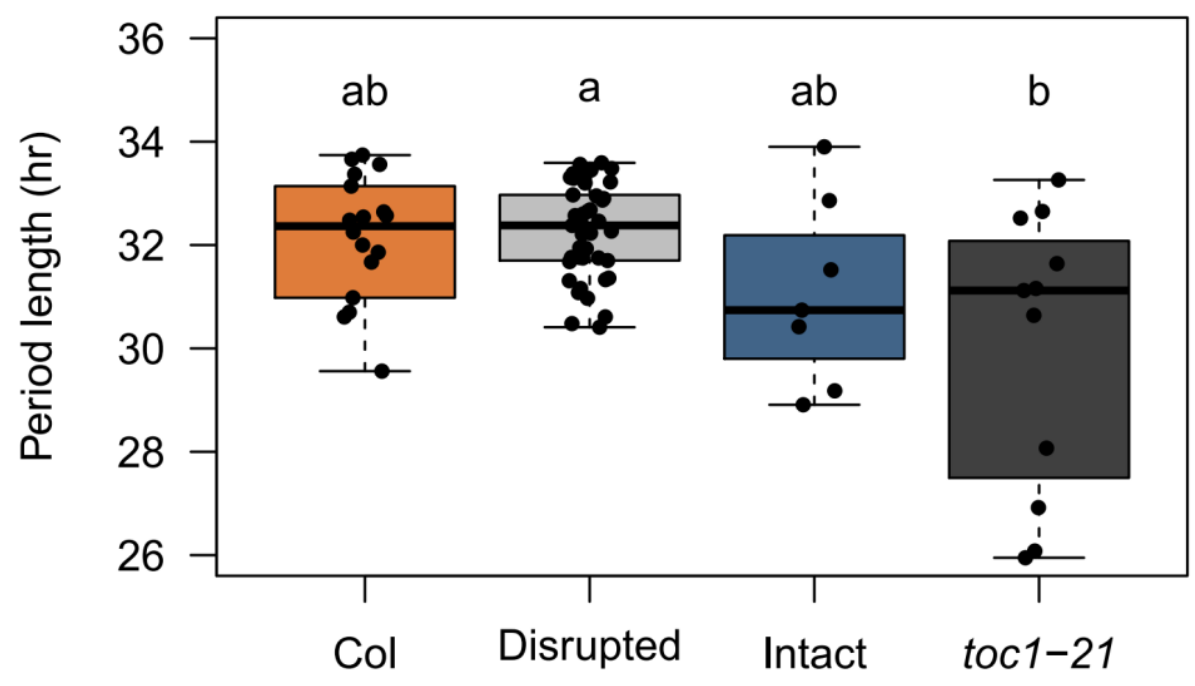

Rhizosphere microbiome treatment

Figure 3: Rhizosphere microbiome treatment leads to clock deceleration in $z t l-1$. The circadian

260 period of $z t l-1$ replicates was significantly shortened when grown with rhizosphere microbial

261 inoculate shaped by plants with short $(21-\mathrm{hr}$, toc $1-21)$ periods $(\mathrm{P}=0.016)$ compared to $z t l-1$

262 replicates grown with a disrupted microbiome treatment or with inoculate shaped by plants with

263 a wild-type period (24-hr, Col). The top and bottom of boxes represent the 75 th and 25 th

264 percentiles, respectively. Whiskers represent 1.5 times the interquartile range. Letters denote

265 significant differences based on Tukey’s Honest Significant Differences post hoc comparisons between rhizosphere microbiome treatments. 